\title{
Evaluación de la calidad de vida en pacientes con cáncer terminal*
}

\author{
Drs. MARCELO FONSECA C. ${ }^{1,3,4}$, CARLOS SCHLACK V., ${ }^{2,4}$, \\ EDISON MERA M. ${ }^{1}$, OSCAR MUÑOZ S. ${ }^{3}$, JAVIER PEÑA L. ${ }^{3}$
}

1 Servicio de Cirugía Hospital Ernesto Torres Galdames, Iquique.

2 Servicio de Cirugía Hospital Dr. Gustavo Fricke, Viña del Mar.

3 Servicio de Urgencia Hospital Ernesto Torres Galdames, Iquique.

${ }^{4}$ Escuela de Medicina, Facultad de Medicina, Universidad de Valparaíso. Valparaíso.

Chile.

\section{Abstract}

\section{Assessment of quality of life in patients with terminal cancer}

Introduction: Research on life quality of cancer patients allows giving the attention back to the patient and his/her needs. Materials and Methods: FACT-G survey was performed to 77 terminal cancer patients on the Palliative Care and Pain Relief unit from Iquique's General Hospital. Results: Physical welfare was evaluated with a 21.22 out of a maximum of 28 , social/familiar welfare had a 12.57 over 28 , emotional welfare was 16 over 24 and functional welfare had 17.33 over 28 . Final score, which represents the sum of the four prior divisions, with a scale from 0 to 108 , was $67.62 \%$ of the maximum score. Conclusions: Performance of life quality surveys is feasible for cancer patients, and allows us to make specific interventions on the patient and his/her close ones to improve his/her life quality.

Key words: Life quality, terminal cancer.

\section{Resumen}

Introducción: Los estudios de calidad de vida en pacientes oncológicos permiten devolver la atención al paciente y a sus necesidades. Material y Método: Se realiza la encuesta FACT-G (Functional Assessment of Cancer Treatment) a 77 pacientes con diagnóstico de cáncer terminal pertenecientes al policlínico de cuidados paliativos y alivio del dolor del Hospital Ernesto Torres Galdames de Iquique. Resultados: El bienestar físico fue evaluado con 21,22 sobre un máximo de 28, el bienestar Social/Familiar obtuvo 12,57 de 28; el bienestar emocional obtuvo 16 sobre 24; el bienestar funcional obtuvo 17,33 sobre 28. El Score final, que representa la suma de las cuatro divisiones anteriores, con una escala de 0 a 108 , fue de 67 , que representa el $62 \%$ del puntaje máximo. Conclusiones: Es factible la realización de encuestas de calidad de vida en pacientes con cáncer, las cuales nos permiten realizar intervenciones puntuales sobre el paciente y sus cercanos que mejoren su calidad de vida.

Palabras clave: Calidad de vida, cáncer terminal.

*Recibido el 7 de marzo de 2013 y aceptado para publicación el 15 de abril de 2013.

Los autores no refieren conflictos de interés

Correspondencia: Dr. Marcelo Fonseca C.

Chipana 2040, Dpto 503, Iquique, Chile.

mfon777@hotmail.com 


\section{Introducción}

No deseo pasar por el final que me espera. Los doctores me indicaron un tratamiento de quimioterapia para vivir un par de años en condiciones razonables. La sola mención de esa palabra insípida me llenó de molestia. ¿Quién más que yo podía juzgar lo que era "razonable"?

(Pablo Simonetti, Madre que estás en los cielos).

Históricamente la evaluación de los tratamientos oncológicos se ha centrado en variables biomédicas, fundamentalmente la tasa de respuesta, el intervalo libre de enfermedad o la supervivencia ${ }^{1,2}$. La disconformidad con el modelo biomédico tradicional ha creado la necesidad de aproximaciones más holísticas, ampliando el concepto de salud a la totalidad de la vida del paciente, sin restringirse sólo a las manifestaciones biológicas ${ }^{2,3}$. La aparición de criterios de valoración dependientes del paciente, resalta aún más este cambio de paradigma ${ }^{4}$. Actualmente, no es adecuado basarse sólo en datos objetivos que reflejen el punto de vista del personal médico, también deben desarrollarse conceptos y herramientas de medición que capten de una forma más amplia y fiable los factores que influyan en el resultado final desde la perspectiva del paciente ${ }^{5}$. El concepto de calidad de vida relacionada con salud, se define como la suma de factores físicos, emocionales y sociales que contribuyen al bienestar de una persona ${ }^{6,7}$. Mediciones de la calidad de vida deberían tener un papel cada vez más importante en la evaluación de la calidad de atención médica ${ }^{8}$.

El presente trabajo es una investigación de tipo exploratorio/descriptivo cuyo objetivo general es evaluar la percepción de la calidad de vida y la factibilidad técnica de evaluarla, a través de la encuesta FACT-G, a pacientes oncológicos terminales ${ }^{10}$, hombres y/o mujeres adultos (as) mayores de 18 años con cáncer, los cuales reciben control y tratamiento en el policlínico de cuidados paliativos y alivio del dolor del Hospital Dr. Ernesto Torres Galdames de Iquique.

\section{Material y Método}

Desde el 1 de mayo de 2010 al 30 de abril de 2011, se realizó la encuesta FACT-G ${ }^{11}$ (Anexo 1a,1b,1c,1d) a los pacientes con diagnóstico de cáncer terminal pertenecientes al policlínico del dolor y cuidados paliativos del Hospital Regional de Iquique. Esto con el consentimiento de la dirección del Hospital y la cooperación del personal médico y paramédico de dicho policlínico. El FACT-G es una recopilación de 27 preguntas generales divididas en cuatro subescalas principales: bienestar físico, bienestar familiar y social, bienestar emocional y bienestar funcional (capacidad de realizar tareas), con un puntaje individual de 0 a 4 . La encuesta fue realizada por el autor y/o otros tres entrevistadores (médicos generales) en forma verbal y llenando además una ficha preestablecida con los datos demográficos y propios del cáncer de cada persona. En los casos en que el paciente no podía acudir al policlínico y el grupo de entrevistadores no pudo concretar una entrevista en domicilio, la entrevista se realizó telefónicamente, siguiendo la misma rutina que en la encuesta efectuada cara a cara. En promedio la entrevista duró aproximadamente $15 \mathrm{~min}$; todas las encuestas fueron hechas con el consentimiento previo de cada paciente. Posterior a esto se confeccionó una base de datos en Excel, la cual fue analizada siguiendo las indicaciones proporcionadas por el grupo FACIT.org ${ }^{12}$.

\section{Resultados}

Desde el 1 de mayo de 2010 al 30 de abril de 2011, se entrevistaron 77 pacientes con diagnóstico de cáncer de diversas etiologías en etapa terminal.

\section{Sexo}

El grupo correspondió a 30 mujeres y 47 varones.

\section{Edad}

El promedio de edad fue de 59 años, con un rango etario desde los 24 a 87 años.

\section{Etiología}

Los tipos de cánceres más frecuentes son: Próstata $(11,6 \%)$, Colon y Recto $(11,6 \%)$, Gástrico $(10,3 \%)$, Pulmón $(9,1 \%)$ y Mama $(7,8 \%)$ (Tabla 1$)$.

Tabla 1. Frecuencia de cáncer

\begin{tabular}{|c|c|}
\hline Cáncer & Frecuencia \\
\hline Próstata & $9(11,6 \%)$ \\
\hline Colon y recto & $9(11,6 \%)$ \\
\hline Gástrico & $8(10,3 \%)$ \\
\hline Pulmón & $7 \quad(9,1 \%)$ \\
\hline Mama & $6 \quad(7,8 \%)$ \\
\hline Cérvico-uterino & $5 \quad(6,50 \%)$ \\
\hline Riñón & $4 \quad(5,20 \%)$ \\
\hline Tiroides & $3(3,90 \%)$ \\
\hline Testicular & $3(3,90 \%)$ \\
\hline Hígado & $3(3,90 \%)$ \\
\hline Páncreas & $2(2,60 \%)$ \\
\hline Vesícula & $2(2,60 \%)$ \\
\hline Otros & $16(20,77 \%)$ \\
\hline
\end{tabular}




\section{Estadios (según clasificación TNM)}

Sesenta y ocho pacientes, equivalentes al $88 \%$ de los pacientes correspondían a cáncer estadio IV; 5 pacientes, equivalentes al $6,5 \%$ del total correspondían a estadio III B, 3 pacientes, equivalentes al 3,9\% del total correspondían a estadio III A y 1 paciente correspondía a estadio I (ingresada para el manejo del dolor) (Tabla 2).

\section{Tratamiento}

Del total de pacientes encuestados (77), las opciones de tratamiento fueron: cirugía, quimioterapia y radioterapia, en forma exclusiva o en combinaciones de las mismas. Veintidós pacientes no recibieron ninguno de los tratamientos antes mencionados, 17 pacientes cirugía exclusiva, 13 pacientes se sometieron a cirugía, quimioterapia y radioterapia, 9 pacientes se sometieron a cirugía más quimiotera-

Tabla 2. Frecuencia de estadios. Según clasificación TNM

\begin{tabular}{|c|c|}
\hline Estadio & Frecuencia \\
\hline I & $1 \quad(1,3 \%)$ \\
\hline II & 0 \\
\hline III A & $3(3,9 \%)$ \\
\hline III B & $5(6,5 \%)$ \\
\hline IV & $68(88,3 \%)$ \\
\hline
\end{tabular}

Tabla 3. Opciones de tratamiento oncológico

\begin{tabular}{|lr|}
\hline Tratamiento & Porcentaje \\
Nada & $22(28,5 \%)$ \\
\hline Cirugía & $17(22,1 \%)$ \\
Quimioterapia & $9(11,7 \%)$ \\
Radioterapia & $3(3,9 \%)$ \\
Cirugía + quimioterapia & $9(11,7 \%)$ \\
Quimioterapia + radioterapia & $4(5,2 \%)$ \\
Cirugía + quimioterapia + radioterapia & $13(16,9 \%)$ \\
\hline
\end{tabular}

pia, 9 pacientes recibieron quimioterapia en forma exclusiva, 4 pacientes se sometieron a quimioterapia y radioterapia y 3 pacientes recibieron radioterapia en forma exclusiva (Tabla 3 ).

\section{Bienestar físico}

Esta división tiene una escala de puntajes de 0 a 28 , siendo mayor la calidad de vida mientras mayor es el resultado numérico. El resultado corregido obtenido es de 21,22 (equivalentes al 76\% del puntaje máximo). La influencia del bienestar físico en la calidad de vida global, teniendo como respuestas de 0 a 10 ( 0 equivale a nada y 10 significa mucho) fue de 5,37 (Tabla 4).

\section{Bienestar social/familiar}

El resultado obtenido es de 12,57 (equivalentes al 45\% del puntaje máximo), siendo de 3,24 la influencia registrada del bienestar social y familiar en la calidad de vida global (Tabla 5).

\section{Bienestar emocional}

Esta división tiene una escala de 0 a 24 (se compone de 6 preguntas en comparación al resto que tiene 7 preguntas por ítems). El resultado obtenido es de 16 (equivalentes al 67\% del puntaje máximo), con una influencia en la calidad de vida de 5,11. En relación a la pregunta "me preocupa que mi enfermedad empeore" no fue incluida en la encuesta por problemas de transcripción, procediéndose a prorratear los resultados tal como lo indica la guía de administración y revisión de la encuesta (Tabla 6).

\section{Bienestar funcional}

Tiene una escala de 0 a 28. Se obtuvo un puntaje final de 17,33 (equivalentes al 62\% del puntaje máximo); con una influencia en la calidad de vida global de 5,6 (Tabla 7).

\section{FACT-G score}

El score final, que representa la suma de las cuatro divisiones anteriores, con una escala de 0 a 108 , fue de 67 , que representa el $62 \%$ del puntaje máximo (Tabla 8).

Tabla 4. Resultados por preguntas sub escala física

\begin{tabular}{|c|c|c|c|c|c|c|c|}
\hline & $\begin{array}{c}\text { Nada } \\
(\%)\end{array}$ & $\begin{array}{c}\text { Un poco } \\
(\%)\end{array}$ & $\begin{array}{l}\text { Algo } \\
(\%)\end{array}$ & $\begin{array}{c}\text { Mucho } \\
(\%)\end{array}$ & $\begin{array}{c}\text { Muchísimo } \\
(\%)\end{array}$ & $\begin{array}{l}\text { NC } \\
(\%)\end{array}$ & Promedio \\
\hline Falta energía & 16,9 & 32,5 & 19,5 & 24,7 & 6,5 & 0 & 2,28 \\
\hline Náuseas & 83,1 & 9,0 & 5,2 & 0 & 2,6 & 0 & 3,70 \\
\hline Atender necesidades familia & 46,7 & 18,2 & 9,1 & 14,3 & 11,7 & 0 & 2,74 \\
\hline Dolor & 28,5 & 35,1 & 16,9 & 18,2 & 1,3 & 0 & 2,71 \\
\hline Efectos secundarios & 84,4 & 9,1 & 0 & 6,5 & 0 & 0 & 3,71 \\
\hline Sentimiento de enfermedad & 37,7 & 32,5 & 15,6 & 9,1 & 2,6 & 2,6 & 2,96 \\
\hline Tiempo acostado & 40,3 & 27,3 & 11,7 & 11,7 & 6,5 & 2,6 & 2,81 \\
\hline
\end{tabular}


Tabla 5. Resultados por preguntas sub escala social/familiar

\begin{tabular}{|lccccccc|}
\hline & $\begin{array}{c}\text { Nada } \\
(\mathbf{\%})\end{array}$ & $\begin{array}{c}\text { Un poco } \\
\mathbf{( \% )}\end{array}$ & $\begin{array}{c}\text { Algo } \\
\mathbf{( \% )}\end{array}$ & $\begin{array}{c}\text { Mucho } \\
\mathbf{( \% )}\end{array}$ & $\begin{array}{c}\text { Muchísimo } \\
\mathbf{( \% )}\end{array}$ & $\begin{array}{c}\text { NC } \\
\mathbf{( \% )}\end{array}$ & Promedio \\
Cercanía amigos & 63,6 & 18,2 & 3,9 & 11,7 & 0 & 2,6 & 0,62 \\
Apoyo emocional familia & 6,5 & 6,5 & 16,9 & 18,2 & 51,9 & 0 & 3,02 \\
Apoyo amigos & 36,3 & 18,2 & 6,5 & 24,7 & 14,3 & 0 & 1,62 \\
Aceptación Ca familia & 6,5 & 1,3 & 27,3 & 42,8 & 22,1 & 0 & 2,72 \\
Satisfacción familia & 54,5 & 22,1 & 10,4 & 11,7 & 0 & 1,3 & 0,79 \\
Cercanía pareja & 5,2 & 0 & 1,3 & 16,8 & 33,8 & 42,9 & 3,29 \\
Vida sexual & 41,5 & 10,4 & 13 & 5,2 & 1,3 & 28,6 & 0,8 \\
\hline
\end{tabular}

Tabla 6. Resultados sub escala emocional

\begin{tabular}{|lccccccc|}
\hline & $\begin{array}{c}\text { Nada } \\
\mathbf{( \% )}\end{array}$ & $\begin{array}{c}\text { Un poco } \\
\mathbf{( \% )}\end{array}$ & $\begin{array}{c}\text { Algo } \\
\mathbf{( \% )}\end{array}$ & $\begin{array}{c}\text { Mucho } \\
\mathbf{( \% )}\end{array}$ & $\begin{array}{c}\text { Muchísimo } \\
\mathbf{( \% )}\end{array}$ & $\begin{array}{c}\text { NC } \\
\mathbf{( \% )}\end{array}$ & Promedio \\
Tristeza & 19,5 & 49,3 & 5,2 & 13 & 13 & 0 & 2,49 \\
Enfrentamiento cáncer & 6,5 & 9,1 & 20,8 & 46,8 & 16,9 & 0 & 2,58 \\
Esperanza/lucha & 46,8 & 23,4 & 15,6 & 14,3 & 0 & 0 & 1,42 \\
Nerviosismo & 37,7 & 23,4 & 13 & 6,5 & 19,5 & 0 & 2,54 \\
Preocupación muerte & 61 & 6,5 & 9,1 & 2,6 & 19,5 & 0 & 2,87 \\
\hline
\end{tabular}

Tabla 7. Resultados sub escala funcional

\begin{tabular}{|lccccccc|}
\hline & $\begin{array}{c}\text { Nada } \\
(\mathbf{\%})\end{array}$ & $\begin{array}{c}\text { Un poco } \\
\mathbf{( \% )}\end{array}$ & $\begin{array}{c}\text { Algo } \\
\mathbf{( \% )}\end{array}$ & $\begin{array}{c}\text { Mucho } \\
\mathbf{( \% )}\end{array}$ & $\begin{array}{c}\text { Muchísimo } \\
\mathbf{( \% )}\end{array}$ & $\begin{array}{c}\text { NC } \\
(\mathbf{\%})\end{array}$ & Promedio \\
\hline Capacidad de trabajo & 24,7 & 14,3 & 35,1 & 18,2 & 7,8 & 0 & 1,7 \\
Satisfacción del trabajo & 14,3 & 6,5 & 7,8 & 48 & 23,4 & 0 & 2,6 \\
\hline Disfrutar la vida & 2,6 & 9,1 & 11,7 & 41,6 & 35 & 0 & 2,97 \\
Aceptación de Ca & 15,6 & 3,9 & 16,9 & 49,3 & 14,3 & 0 & 2,43 \\
Buen Sueño & 5,2 & 26 & 19,5 & 37,7 & 11,7 & 0 & 2,25 \\
Disfrutar pasatiempos & 5,2 & 5,2 & 10,4 & 45,4 & 33,8 & 0 & 2,97 \\
Satisfacción calidad vida & 16,9 & 3,9 & 22,1 & 38,9 & 13 & 5,2 & 2,29 \\
\hline
\end{tabular}

Tabla 8. Tabla Resumen Encuesta FACT-G

\begin{tabular}{|ccccccccc|}
\hline Física & CV & Social/familiar & CV & Emocional & CV & Funcional & CV & FACT-G score \\
21,22 & 5,37 & 12,57 & 3,24 & 16 & 5,11 & 17,33 & 5,6 & 67 \\
\hline
\end{tabular}

\section{Discusión}

El estudio de la calidad de vida en pacientes oncológicos ha experimentado últimamente un gran auge, debido al reconocimiento de que muchos cánceres avanzados son incurables, lo cual ha llevado a potenciar los cuidados paliativos ${ }^{13}$. Muchos pacientes, al saber su condición, suelen estar más interesados en no perder su autonomía física y en padecer el menor número de síntomas, que en la prolongación de la vida (a veces a costa de mucha toxicidad $^{14,15}$. Por otro lado, la escasez de recursos económicos justifica únicamente la incorporación de nuevos fármacos que, si no incrementan la supervivencia, al menos produzcan un beneficio sintomático apreciable $^{16}$. 
Para medir la calidad de vida en este grupo de pacientes se utilizó la encuesta FACT-G (Functional Assessment of Cancer Treatment), que como ya se mencionó, es un grupo de escalas basadas en un cuestionario principal de 27 preguntas, subdivididas en cuatro ámbitos: físico, funcional, social/familiar y emocional.

El bienestar físico es evaluado a través de preguntas sobre: estado de energía, presencia de náuseas, dolor o efectos secundarios del tratamiento, capacidad de atender las necesidades de la familia, conciencia de enfermedad y necesidad de permanecer acostado debido al cáncer. Destaca el buen puntaje obtenido 21,22 de 28 puntos máximos; de las cuatro sub escalas valoradas ésta es la con mayor puntaje; la importancia del bienestar físico en la calidad de vida global es de 5,37. La falta de energía muestra un promedio de respuestas corregido de 2,28 , lo cual está de acuerdo con la literatura que muestra la gran prevalencia de falta de energía o cansancio crónico (dentro de las múltiples denominaciones). Se ha informado que el cansancio crónico relacionado con el tratamiento del cáncer está presente entre 14 y $96 \%$ de los pacientes que se encuentran en tratamiento ${ }^{17}$ y entre 19 y $82 \%$ de los pacientes después del tratamiento ${ }^{18}$, teniendo un origen multifactorial. En este grupo los pacientes refieren muy pocas náuseas $(3,70 / 4)$ y efectos secundarios del tratamiento $(3,71 / 4)$, probablemente por el estricto control en la unidad de cuidados paliativos. Las náuseas y vómitos pueden provocar serios trastornos metabólicos, deterioro nutricional y anorexia, deterioro del estado mental y físico del paciente, desgarros esofágicos, dehiscencia de heridas, interrupción del tratamiento antineoplásico y alteraciones de la capacidad funcional ${ }^{19}$. El $80 \%$ de los pacientes con cáncer va a presentar algún tipo de dolor a lo largo de su enfermedad $^{20}$. En nuestro estudio el manejo del dolor se calificó como bueno $(2,72 / 4)$.

Este estudio demuestra que la calidad de vida en los pacientes con cáncer se ve más afectada en el ámbito social y familiar (12,57/28). Sin embargo, al preguntar cuánto afecta el aspecto social y familiar a la calidad de vida de los pacientes se obtiene una baja influencia $(3,24 / 10)$, quizás porque el miedo al dolor y a la muerte puede interferir en la vida social hasta el punto de reducirla a mínimos. La evidencia asocia el aislamiento y la carencia de conexiones sociales al aumento de riesgo en la morbilidad y mortalidad $^{21}$. Se acepta que el sistema social puede ayudar a individuos a resistir los efectos psicológicos adversos de la severa tensión causada por la enfermedad, promoviendo una mejor recuperación física y mental ${ }^{22}$. La valoración de la cercanía de las amistades es menos que "algo" $(1,62 / 4)$, lo que refleja el grado de aislamiento adquirido o autoimpuesto en esta etapa terminal. Destaca la lejanía $(0,62 / 4)$ y mala comunicación $(0,79 / 4)$ con la familia de parte del paciente con cáncer, pero sin embargo, existe un sentimiento de apoyo $(3,02 / 4)$ y aceptación $(2,7 / 4)$. "Están, me ayudan, pero no entienden". El cáncer es una enfermedad que tiene la particularidad de envolver y sobrecoger a toda la familia, cambiando las rutinas y modificando hasta detalles de la vida cotidiana $^{22}$. La pregunta sobre la cercanía con la pareja o a la persona que es la principal fuente de apoyo fue la que obtuvo la mayor tasa de omisiones (33/77; equivalentes al $42,9 \%$ ), lo que quizás refleja un alto grado de lejanía del paciente terminal con las parejas respectivas. Sin embargo, de las personas que contestaron la pregunta el grado de cercanía es alto $(3,29 / 4)$. Los datos sobre el ajuste y la supervivencia demuestran que gente casada de diferente edad y de ambos géneros se adapta mejor a la enfermedad crónica y tiene una calidad de vida más elevada que gente soltera ${ }^{22}$. La satisfacción sexual en este estudio es muy baja, con un puntaje de 0,8 de un máximo de 4 , asociándose además a un importante número de omisión de la pregunta (22/77), teniendo en cuenta que en el encabezado de la pregunta recalcaba la no importancia de la actividad sexual actual. Síntomas y consecuencias de la enfermedad, como la anemia y la anorexia, pueden provocar debilidad y dificultar la actividad sexual. Otras veces los tratamientos utilizados (drogas, radioterapia, intervenciones quirúrgicas) pueden producir efectos similares y hasta limitar considerablemente las relaciones sexuales. Los factores de orden psicológico (depresión, ansiedad) tienen gran importancia en la aparición de dificultades de orden sexual. Ciertas secuelas quirúrgicas pueden producir trastornos de la imagen corporal, sentimientos de desagrado hacia sí mismo y hacia la pareja. El estadio terminal por sí mismo no disminuye necesariamente el interés sexual, por el contrario, puede acrecentarse la necesidad de cercanía física y de ser reconocida su condición de ser humano sexualmente deseable, aun cuando sea totalmente imposible la realización del coito ${ }^{23,24}$.

El puntaje que refleja el bienestar emocional fue de $67 \%$, con una influencia sobre la calidad de vida de 5,1. La tristeza y la pena son reacciones normales al diagnóstico de cáncer, por lo tanto, es importante distinguir entre los grados normales de tristeza y los trastornos depresivos.

El bienestar funcional fue evaluado con 17,33/28, con una influencia en la calidad de vida de 5,61/10. El bienestar funcional tiene mucha relación a en que etapa del proceso de adaptación se encuentre. Los pacientes portadores de cáncer parecen atravesar generalmente una serie predecible de etapas emocionales clásicas a veces difíciles de distinguir, que varían individualmente en duración e intensidad ${ }^{25}$. Los 
trastornos del sueño están presentes en esta serie, el puntaje obtenido es de 2,24/4. Los pacientes con cáncer tienen gran riesgo de desarrollar insomnio y trastornos del ciclo sueño-vigilia. El insomnio es el trastorno del sueño más común en esta población y con mayor frecuencia suele ser secundario a los factores físicos y psicológicos relacionados con el cáncer y su tratamiento. La ansiedad y la depresión, respuestas psicológicas comunes al diagnóstico de cáncer, están sumamente correlacionadas con el insomnio ${ }^{26}$.

\section{Conclusiones}

Las encuestas de calidad de vida son sencillas de realizar y nos permiten principalmente devolver la atención en la persona y sus necesidades, permi- tiéndonos entregarles lo que ellas necesitan y no lo que los médicos suponen. Estas debieran ser incorporadas a la evaluación rutinaria y periódica de los pacientes oncológicos, ya que permiten pesquisar oportunamente las áreas problemáticas, y en un segundo tiempo realizar intervenciones puntuales no sólo en el ámbito médico, que le brinden al paciente, familia y amigos una mejor calidad de vida en su etapa terminal.

\section{Agradecimientos}

Al personal médico, paramédico y administrativo del policlínico de Cuidados paliativos y alivio del dolor del Hospital Ernesto Torres Galdames de Iquique.

Anexo 1.a.

\begin{tabular}{|c|c|c|c|c|c|}
\hline Estado físico general de salud & Nada & Un poco & Algo & Mucho & Muchísimo \\
\hline Me falta energía & 0 & 1 & 2 & 3 & 4 \\
\hline Tengo náuseas & 0 & 1 & 2 & 3 & 4 \\
\hline $\begin{array}{l}\text { Debido a mi estado físico, tengo dificultad para atender las necesi- } \\
\text { dades de mi familia }\end{array}$ & 0 & 1 & 2 & 3 & 4 \\
\hline Tengo dolor & 0 & 1 & 2 & 3 & 4 \\
\hline Me molestan los efectos secundarios del tratamiento & 0 & 1 & 2 & 3 & 4 \\
\hline Me siento enfermo(a) & 0 & 1 & 2 & 3 & 4 \\
\hline Tengo que pasar tiempo acostado(a) & 0 & 1 & 2 & 3 & 4 \\
\hline
\end{tabular}

Anexo 1.b.

\begin{tabular}{|c|c|c|c|c|c|}
\hline Ambiente familiar y social & Nada & Un poco & Algo & Mucho & Muchísimo \\
\hline Me siento cercano(a) a mis amistades & 0 & 1 & 2 & 3 & 4 \\
\hline Recibo apoyo emocional por parte de mi familia & 0 & 1 & 2 & 3 & 4 \\
\hline Recibo apoyo por parte de mis amistades & 0 & 1 & 2 & 3 & 4 \\
\hline Mi familia ha aceptado mi enfermedad & 0 & 1 & 2 & 3 & 4 \\
\hline $\begin{array}{l}\text { Estoy satisfecho(a) con la manera en que se comunica mi familia } \\
\text { acerca de mi enfermedad }\end{array}$ & 0 & 1 & 2 & 3 & 4 \\
\hline $\begin{array}{l}\text { Me siento cercano(a) a mi pareja (o la persona que es mi principal } \\
\text { fuente de apoyo) }\end{array}$ & 0 & 1 & 2 & 3 & 4 \\
\hline \multicolumn{6}{|c|}{$\begin{array}{l}\text { Sin importar su nivel actual de actividad sexual, conteste a la siguiente pregunta. Si prefiere no contestarla, omitala y siga } \\
\text { con la siguiente sección }\end{array}$} \\
\hline Estoy satisfecho con mi vida sexual & 0 & 1 & 2 & 3 & 4 \\
\hline
\end{tabular}


Anexo 1.c.

\begin{tabular}{|l|c|c|c|c|c|}
\hline Estado emocional & Nada & Un poco & Algo & Mucho & Muchísimo \\
\hline Me siento triste & 0 & 1 & 2 & 3 & 4 \\
\hline Estoy satisfecho de cómo me estoy enfrentando a mi enfermedad & 0 & 1 & 2 & 3 & 4 \\
\hline Estoy perdiendo las esperanzas en la lucha contra mi enfermedad & 0 & 1 & 2 & 3 & 4 \\
\hline Me siento nervioso(a) & 0 & 1 & 2 & 3 & 4 \\
\hline Me preocupa morir & 0 & 1 & 2 & 3 & 4 \\
\hline Me preocupa que mi enfermedad empeore & 0 & 1 & 2 & 3 & 4 \\
\hline
\end{tabular}

Anexo 1.d

\begin{tabular}{|l|c|c|c|c|c|}
\hline Capacidad de funcionamiento personal & Nada & Un poco & Algo & Mucho & Muchísimo \\
\hline Puedo trabajar (incluya trabajo en el hogar) & 0 & 1 & 2 & 3 & 4 \\
\hline Mi trabajo me satisface (incluya el trabajo en el hogar) & 0 & 1 & 2 & 3 & 4 \\
\hline Puedo disfrutar de la vida & 0 & 1 & 2 & 3 & 4 \\
\hline He aceptado mi enfermedad & 0 & 1 & 2 & 3 & 4 \\
\hline Duermo bien & 0 & 1 & 2 & 3 & 4 \\
\hline Disfruto con mis pasatiempos de siempre & 0 & 1 & 2 & 3 & 4 \\
\hline Estoy satisfecho(a) con mi calidad de vida actual & 0 & 1 & 2 & 3 & 4 \\
\hline
\end{tabular}

\section{Referencias}

1. Karnofsky DA, Burchenal JH. The clinical evaluation of chemotherapeutic agents in cancer. En: CM Macleod (Ed.). Evaluation of chemotherapeutic agents. New York: Columbia University Press, 1949;191-205.

2. Oliver A, Greenberg C, Evaluación de resultados en el tratamiento oncológico: importancia de los criterios de valoración dependientes del paciente. Surg Clin N Am. 2009;89:17-25.

3. Taboada P. Introducción a la monografía: El derecho al bien morir y el concepto de muerte digna. Ars Medica. Revista de Estudios médico humanísticos. Vol. 5. $\mathrm{N}^{\circ} 5$. Noviembre 2001. Disponible en: http://escuela.med. puc.cl/publ/arsmedica/arsmedica2/02_Taboada.html (Consultado el 7 de marzo de 2011).

4. Taboada P. El derecho a morir con dignidad. Acta bioeth. 2000;6:89-101.

5. Bottomley A, Aaronson NK. European Organisation for Research and Treatment of Cancer. International perspective on health-related quality-of-life research in cancer clinical trials: theEuropean Organisation for Research and Treatment of Cancer experience. J Clin Oncol. 2007;25:5082-6.

6. Moinpour CM, Feigl P, Metch B. Quality of life end points in cancer clinical trials: review and recommendations. J Natl Cancer Inst. 1989;81:485-95.
7. Badia X, García F. La medición de la calidad de vida relacionada con la salud y las preferencias en estudios de investigación de resultados en salud. En Badia, X. (Ed.) La Investigación de Resultados en Salud. De la evidencia a la práctica clínica. Barcelona, Edimac 2000.

8. Font A. Cáncer y calidad de vida. Anuario de Psicología, Universitat de Barcelona. 1994;61:41-50.

9. Simonetti P, Madre que estás en los cielos. Editorial Planeta Chile SA. Decimocuarta edición, diciembre de 2009.

10. MINSAL. Norma técnica $n^{\circ} 31$ y 32, programa alivio del dolor y cuidados paliativos.

11. FACIT Administration and Scoring Guidelines. www. facit.org

12. FACT-G Scoring Guidelines. Version 4. www.facir.org

13. Guerrero M. La paliación en los albores del nuevo siglo. Tema monográfico: Cuidados paliativos en el enfermo terminal. Ars Medica 2005;11:11-3.

14. Piñera B. El médico y la muerte. Ars Medica Vol. 2. $\mathrm{N}^{\circ}$ 2. Mayo de 2000. Disponible en: http://escuela. med.puc.cl/publ/arsmedica/ArsMedica2/07_Pinera.html (Consultado el 1 de febrero de 2011).

15. Bouësseau M. La muerte como frontera del sentido: Fundamentos para la elaboración de una ética de la medicina paliativa. Ars Medica Vol. 2. N². Mayo de 2000. Disponible en: http://escuela.med.puc.cl/publ/ars- 
medica/ArsMedica2/08 Bouesseau.html. (Consultado el 2 de febrero de 2011).

16. Cella DF, Tulsky DS, Gray C. The functional assessment of cancer therapy scale: development and validation of the general measure. J Clin Oncol. 1993;11: $570-9$.

17. Miaskowski C, Portenoy RK. Update on the assessment and management of cancer-related fatigue. Principles and Practice of Supportive Oncology Updates 1998;1:110.

18. Prue G, Rankin J, Allen J, Gracey J, Cramp F. Cancerrelated fatigue: A critical appraisal. Eur $\mathrm{J}$ Cancer 2006;42:846-63.

19. Craig JB, Powell BL. The management of nausea and vomiting in clinical Oncology. Am J Med Sci. 1987;293:34-44.

20. Moreira JS, Sánchez FD. Evaluación del dolor en cuidados paliativos. En: Guías Clínicas de Cuidados Paliativos. Sociedad Española de Cuidados Paliativos 2007.

21. Hann D, Baker F, Denniston M, Gesme D, Reding $\mathrm{D}$, Flynn T, et al. The influence of social support on depressive symptoms in cancer patients' age and gender differences. Journal of Psychosomatic Research 2002;52:279-83.

22. Baider L. Cáncer y familia: aspectos teóricos y terapéuticos. Revista Internacional de Psicología Clínica y de la Salud. 2003;3:505-20.

23. Derogatis LR, Kourlesis SM: An approach to evaluation of sexual problems in the cancer patient. CA Cancer J Clin. 1981;31:46-50.

24. Grau J, Llanta M, Chacón M. La sexualidad en pacientes con cáncer: algunas consideraciones sobre su evaluación y tratamiento. Rev Cubana Oncol. 1999;15:49-65.

25. Wilson KG, Chochinov HM, Skirko MG, Allard P, Chary S, Gagnon PR, et al. Depression and anxiety disorders in palliative cancer care. J Pain Symptom Manage 2007;33:118-29.

26. Engstrom CA, Strohl RA, Rose L, Lewandowski L, Stefanek ME. Sleep alterations in cancer patients. Cancer Nursing 1999;22:143-8.

27. Medina E, Kaempffer Ana. Mortalidad del adulto en Chile. Rev Med Chile 2000;128:1144-9.

28. Fulton R. La negación de la muerte. En una antología: La muerte y el morir; desafío y cambio. Fondo Educativo Interamericano Incorporado. 1981.
29. Reyes M, Santi B, Nervi F. Cuidados paliativos y control de síntomas en Chile. La experiencia de la Clínica Familia. Ars Medica 2005;11:187-93.

30. Lolas F. Evitar la distancia tecnocrática. Objetivos de los cuidados paliativos. Ars Medica. Revista de Estudios médico humanísticos. 2005;11:17-22.

31. Ministerio de Salud de Chile. Guía Clínica Alivio del dolor por Cáncer Avanzado y Cuidados Paliativos.1st Ed. Santiago: Minsal, 2005.

32. Taboada P. Principios éticos relevantes en la atención de pacientes terminales. Ars Medica. Revista de Estudios médico humanísticos. Vol. 12. $\mathrm{N}^{\circ} 12$. Disponible en: http://escuela.med.puc.cl/publ/arsmedica/ArsMedica12/ PrincipiosEticos.html. (Consultado el 7 de febrero de 2011).

33. Astudillo W, Da Rocha C, Mendinueta C. Alivio de las situaciones difíciles y del sufrimiento en la terminalidad. $1^{\text {ra }}$ Edición. San Sebastián, 2005. Sociedad Vasca de Cuidados Paliativos.

34. Fonnegra I,. El médico ante el sufrimiento del paciente que enfrenta su muerte. Ars Medica. Revista de Estudios médico humanísticos. Vol. 3. $\mathrm{N}^{\circ} 3$. Noviembre de 2000. Disponible en: http://escuela.med.puc.cl/publ/ arsmedica/ArsMedica3/03_Fonnegra.html. (Consultado 3 de febrero de 2011).

35. Alonso A, González M. Medidas de Capacidad funcional. En: Valoración clínica en el paciente con cáncer. Editorial Médica Panamericana 2006.

36. Astudillo W, Mendinueta C. ¿Cómo mejorar la comunicación en la fase terminal?. Ars Medica 2005;11:61-85.

37. Franca O, Fontes N, González M, Núñez J, Puig R, Queirolo H, Silva Y. La ayuda (médica) al bien morir. Ars Medica. Revista de Estudios Médico Humanísticos. 2005;11:61-85.

38. Fernández-López J, Fernández-Fidalgo M, Alarcos C. Los conceptos de calidad de vida, salud y bienestar analizados desde la perspectiva de la clasificación internacional del funcionamiento. Rev Esp Salud Pública 2010;84:169-84.

39. Ruiz MA, Pardo A. Calidad de vida relacionada con la salud: Definición y utilización en la práctica médica. Pharmacoeconomics Spanish Research Articles 2005;2:31-43.

40. Schwartzmann L. Calidad de vida relacionada con la salud: Aspectos conceptuales. Ciencia y Enfermería 2003;9:9-21. 